\title{
$\underline{\text { Calculation of PCB Track Impedance }}$
}

\author{
by \\ Andrew J Burkhardt, Christopher S Gregg and J Alan Staniforth
}

\section{INTRODUCTION}

The use of high-speed circuits requires PCB tracks to be designed with controlled (characteristic, odd-mode, or differential) impedances. Wadell ${ }^{[1]}$ is one of the most comprehensive sources of equations for evaluating these impedances. This source includes many configurations including stripline, surface microstrip, and their coplanar variants.

The IPC publication, IPC-2141 $1^{[2]}$, is another source of equations but has a smaller range of configurations, similar to those presented in IPC-D-317A.

However, for some configurations there are differences between the equations given in these publications. The authors believe that it is now opportune to examine the origin of the equations and to update the method of calculation for use with modern personal computers.

As an example, consider the surface microstrip shown in Figure 1.

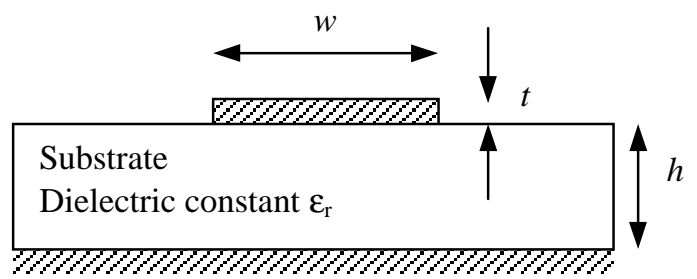

Figure 1 - Surface Microstrip

IPC-2141 ${ }^{[2]}$ gives the characteristic impedance as

$Z_{0}=\frac{87.0}{\left(\varepsilon_{r}+1.41\right)^{1 / 2}} \ln \left[\frac{5.98 \mathrm{~h}}{0.8 w+t}\right]$

Wadell ${ }^{[1]}$ gives

$$
Z_{0}=\frac{\eta_{0}}{2.0 \sqrt{2.0} \pi\left(\varepsilon_{r}+1\right)^{1 / 2}} \ln \left[1.0+\frac{4.0 h}{w^{\prime}}(A+B)^{1 / 2}\right]
$$

where $\quad A=\frac{14.0+8.0 / \varepsilon_{r}}{11.0} \times \frac{4.0 h}{w^{\prime}}$

$$
B=\left(A^{2}+\frac{1.0+1.0 / \varepsilon_{r}}{2.0} \times \pi^{1 / 2}\right)^{1 / 2}
$$

with

$$
w^{\prime}=w+\Delta w^{\prime}
$$

The parameter $w^{\prime}$ is the equivalent width of a track of zero thickness due to a track of rectangular profile, width $w$ and thickness $t$. Wadell ${ }^{[1]}$ gives an additional equation to determine the incremental value $\Delta w^{\prime}$. The parameter $\eta_{o}$, in equation (2), is the impedance of free-space (or vacuum), $376.7 \Omega(\approx 120 \pi)$. The quoted accuracy is $2 \%$ for any value of $\varepsilon_{r}$ and $w$.

Table 1 shows the results of applying equations (1) and (2) to a popular surface microstrip constructed from 1oz copper track on $1 / 32$ inch substrate.

Table 1

\begin{tabular}{|c|c|c|c|c|c|}
\hline \multirow{2}{*}{$\begin{array}{c}\text { Width } \\
w \\
(\mu \mathrm{m})\end{array}$} & \multirow{2}{*}{$\begin{array}{c}\text { Numerical } \\
\text { Method } \\
Z_{0}(\Omega)\end{array}$} & \multicolumn{2}{|c|}{ Equation (1) } & \multicolumn{2}{c|}{ Equation (2) } \\
\cline { 3 - 6 } & $Z_{0}(\Omega)$ & $\%$ error & $Z_{0}(\Omega)$ & $\%$ error \\
\hline 3300 & 30.09 & 21.08 & -29.94 & 29.89 & -0.66 \\
\hline 1500 & 50.63 & 49.46 & -2.31 & 50.50 & -0.26 \\
\hline 450 & 89.63 & 91.79 & +2.41 & 89.89 & +0.29 \\
\hline
\end{tabular}

$$
t=35 \mu \mathrm{m}, h=794 \mu \mathrm{m}, \varepsilon_{r}=4.2
$$

(the calculation of the error assumes the numerical method is accurate : see Numerical Results)

Table 1 shows that equation (2) is well within the quoted accuracy. The accuracy of equation (1) varies widely, but this equation has the advantage of simplicity and is useful in illustrating the general changes to the value of $Z_{0}$ as the width $w$ and thickness $t$ are varied.

The example demonstrated by Table 1 , highlights the general problem with published equations: complicated equations are usually more accurate. Ranges over which the equations are accurate are also usually restricted to a limited range of parameters (e.g. $w / h, t / h$ and $\varepsilon_{r}$ ).

Equation (2) is complicated, but with patience, can be evaluated using a programmable calculator or computer 
spreadsheet. However the complications increase greatly when two coupled tracks are used to give a differential impedance. For coupled surface microstrip, Wadell ${ }^{[1]}$ gives 7 pages of equations to evaluate the impedance.

It is now a major exercise to evaluate the impedance using a calculator or spreadsheet.

\section{ALGEBRAIC EQUATIONS}

\section{Single Track}

For the stripline of Figure 2 with a symmetrically centred track of zero thickness, $\mathrm{Cohn}^{[3]}$ has shown that the exact value of the characteristic impedance is

$$
Z_{0}=\frac{\eta_{0}}{4.0 \sqrt{E_{r}}} \frac{K(k)}{K\left(k^{\prime}\right)}
$$

where

$$
k=\operatorname{sech}\left(\frac{\pi w}{2.0 h}\right)
$$

and

$$
k^{\prime}=\tanh \left(\frac{\pi w}{2.0 h}\right)
$$

$K$ is the complete elliptic function of the first kind ${ }^{[4]}$. An equation for the evaluation of the ratio of the elliptic functions, accurate to $10^{-12}$, has been given by Hilberg ${ }^{[5]}$, and also quoted by Wadell ${ }^{[1]}$.

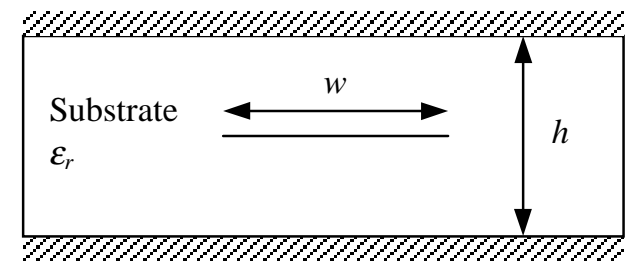

Figure 2 - Stripline: Centred Track

When the thickness is not zero, corrections have to be made which are approximate ${ }^{[1]}$. These corrections are obtained from theoretical approximations or curve fitting the results of numerical calculations based on the fundamental electromagnetic field equations.

When the track is offset from the centre, the published equations become more complicated and the range of validity, for a given accuracy, is reduced.

Attempts have also been made to include the effects of differential etching on the track resulting in a track crosssection which is trapezoidal ${ }^{[1]}$.

There is no closed-form equation like equation (4) for surface or embedded microstrip of any track thickness. Thus any equation used to calculate the impedance is approximate and demonstrated in Table 1.

\section{Coupled Coplanar Tracks}

Figure 3 shows two coupled coplanar centred stripline tracks.

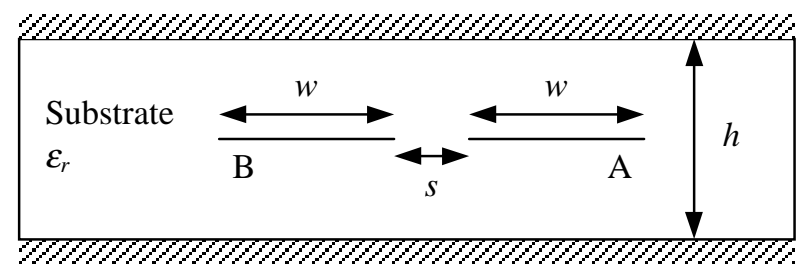

Figure 3 - Stripline : Coplanar
Coupled Centred Tracks

All the impedance equations for coupled configurations refer to both even-mode impedance $\left(Z_{0 e}\right)$ and odd-mode impedance $\left(Z_{0 o}\right)$. These impedances are measured between the tracks and the ground plane. $Z_{0 e}$ occurs when tracks $\mathrm{A}$ and $\mathrm{B}$ are both at $+\mathrm{V}$ relative to the ground plane, and $Z_{0 o}$ occurs when track $A$ is at $+V$ and track $B$ is at $-V$. When a differential signal is applied between $\mathrm{A}$ and $\mathrm{B}$, then a voltage exists between the tracks similar to the odd-mode configuration. The impedance presented to this signal is then the differential impedance,

$Z_{\text {diff }}=2 \times Z_{0 o}$

All published equations [1] give $Z_{0 o}$. The differential impedance must then be obtained using equation (6).

For the zero thickness configuration of Figure 3, Cohn ${ }^{[3]}$ gives the exact expression.

$Z_{0 o}=\frac{\eta_{0}}{4.0 \sqrt{\varepsilon_{r}}} \frac{K\left(k_{0}\right)}{K\left(k_{0}^{\prime}\right)}$

where

$k_{0}=\left(1-k_{0}^{\prime 2}\right)^{1 / 2}$

and

$$
k_{0}^{\prime}=\tanh \left[\frac{\pi w}{2.0 h}\right] \operatorname{coth}\left[\frac{\pi(w+s)}{2.0 h}\right]
$$

As before $K$ is the elliptic function of the first kind. There are no closed-form equations for coplanar coupled tracks.

\section{Effect of Track Thickness}

When the track thickness is not zero, approximations must be made to obtain algebraic equations similar to equations (4) and (7). Alternatively, equations, based on curve fitting of extensive numerical calculations, are used.

However, as the thickness increases the impedance decrease, as can be noted from equation (1). 


\section{NUMERICAL PRINCIPLES}

For pulses on a uniform transmission system, ${ }^{[1,6]}$ then

$Z_{0} \quad\left(\right.$ or $\left.Z_{0 o}\right)=\sqrt{\frac{L}{C}}$

where $L$ is the inductance and $\mathrm{C}$ the capacitance per unit length of line.

For a stripline, where the electric (and magnetic) fields are in a uniform substrate, dielectric constant $\varepsilon_{r}$, equation (9) becomes

$Z_{0}=\frac{\sqrt{\varepsilon_{r}}}{c C}$

where $c$ is the velocity of light in vacuuo (or free-space). The velocity of pulse travel along the transmission path is

$v=\frac{c}{\sqrt{\varepsilon_{r}}}$

For a microstrip, the electric (and magnetic) fields are in air and the substrate, It can be shown that

$Z_{0}=\frac{1}{c \sqrt{C C_{\text {air }}}}$

Where $C_{a i r}$ is the capacitance of the same track configuration without substrate. The effective dielectric constant is

$\varepsilon_{e f f}=\frac{C}{C_{a i r}}$

To find the impedance, the capacitance must be calculated. This can be done by applying a voltage $V$ to the tracks and calculating the total charge per unit length $Q$, from which

$C=\frac{Q}{V}$

However the surface charge on a track is not uniform. In fact it is very high at track corners. Therefore the total charge is difficult to calculate.

From electrostatic theory, it is known that a charge produces a voltage at a distance $r$ from the charge. Then a distribution of charge $\rho$ (coulomb/unit width of track) gives a voltage

$V=\int G \rho \delta l$

where the integral is taken over the perimeter of the track cross-section, $\delta l$ is a small length, and $G$ is the voltage due to a unit charge. It is also known as the Green's Function. The value of $G$ depends on the configuration (or environment). For instance, a point charge in a 2 dimensional dielectric space, without conductors gives

$$
V=-\frac{\rho \ln (r)}{2 \pi \varepsilon_{0} \varepsilon_{r}}
$$

so that $\quad G=-\frac{\ln (r)}{2 \pi \varepsilon_{0} \varepsilon_{r}}$

In equation (15), the voltage $V$ is known, $G$ is known for the particular configuration of tracks and substrate, but the charge $\rho$ is unknown. Thus (15) is an integral equation which can be solved numerically by the Method of Moments (MoM) ${ }^{[7]}$.

To proceed using MoM, the cross-section perimeter of the track is divided into short lengths with a node at each end. Charges are assigned to each node. The voltage at each node is calculated from all the nodal charges and the estimated charge variation between nodes. This leads to a set of simultaneous equations represented by the matrix equation

$$
\mathbf{A} \boldsymbol{\rho}=\mathbf{V}
$$

where $\boldsymbol{\rho}$ is a vector of nodal charges, and $\mathbf{V}$ is a vector of nodal voltages. A is a square matrix whose elements are calculated from integrals involving the Green's Function. The size of the matrices depends on the number of nodes. Equation (17) can be solved for the nodal charges $\rho$ for given nodal voltages $\mathbf{V}$. The elements of $\mathbf{V}$ are usually +1 or -1 depending on the configuration.

The total charge $Q$ can be obtained by a suitable summation of the nodal charges.

This general approach has been used by most authors to evaluate the various impedances. Most of the calculations were published 15 to 20 years ago, when the principal calculator was a main-frame computer. Hence the need for equations which could be used with the pocket calculators available at that time. 
The present authors have revisited the basic numerical approach and have developed software ${ }^{[8]}$ which readily calculates the controlled impedances using a desktop PC. The software runs quickly on a modern PC, and has been extended to also include the calculation of configurations not well represented in the literature. This includes

- offset coupled stripline,

- broadside coupled stripline,

- $\quad$ embedded coupled microstrip.

Thick tracks are normally to be expected which have a trapezoidal cross-section to allow for differential etching of the track.

\section{NUMERICAL RESULTS}

This section describes in more detail some of the numerical techniques and compares the results with the exact equations (4) and (7).

In all cases the Green's Function for the configurations was obtained using charge images in the ground planes. There are an infinite number of these images. In the case of stripline the sum of images converges to the result given by Sadiku $^{[9]}$. Silvester ${ }^{[10,11]}$ developed the image method for surface microstrip and has now been extended by the authors for embedded microstrip. In all cases the sum of images converges, but the result has to be obtained numerically.

The distribution of charge over an element between nodes is assumed to be linear. A numerical singularity occurs when the charge node $j$ coincides with the voltage node $i$. Sadiku $^{[9]}$ indicated how this can be resolved. The evaluation of the elements $A_{i j}$ consists of both numerical and analytic integration in the same manner as that used in Boundary Element techniques ${ }^{[12,13]}$.

To avoid numerical inaccuracies at corners where there is a large concentration of charge, the length of an element at a corner is made very small. The other elements and nodes are then distributed by the method described by Kobayashi $^{[14]}$. This means that wide strips require more nodes than narrow strips when the same small element is used.

The results presented were performed on a PC with an Intel Pentium Pro running at $233 \mathrm{MHz}$ using a compiled Cprogram.

\section{Single Track Stripline}

Figure 4 shows the variation of impedance with track width for the stripline of Figure 2.

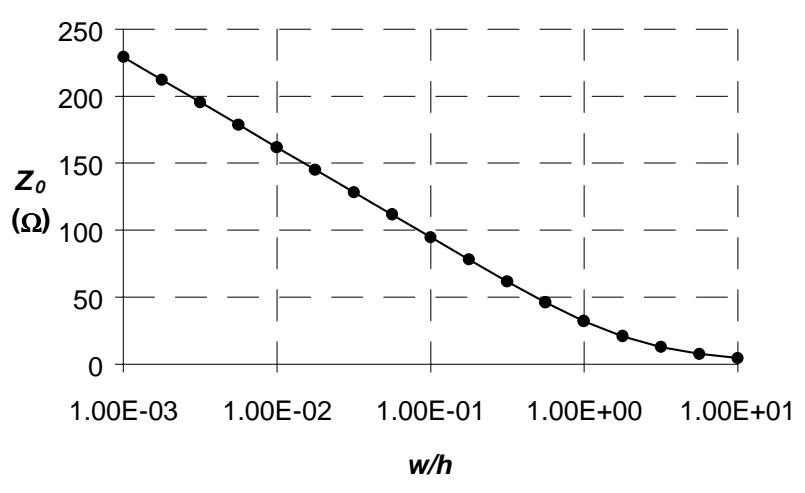

Figure 4 - Impedance for different relative width (Substrate $\varepsilon_{r}=\mathbf{4 . 2}$ )

Figure 5 shows the \% error of the numerical calculation compared with the exact values given by equation (4). Two curves are shown for different small elements at the corner (i.e. ends of the track).

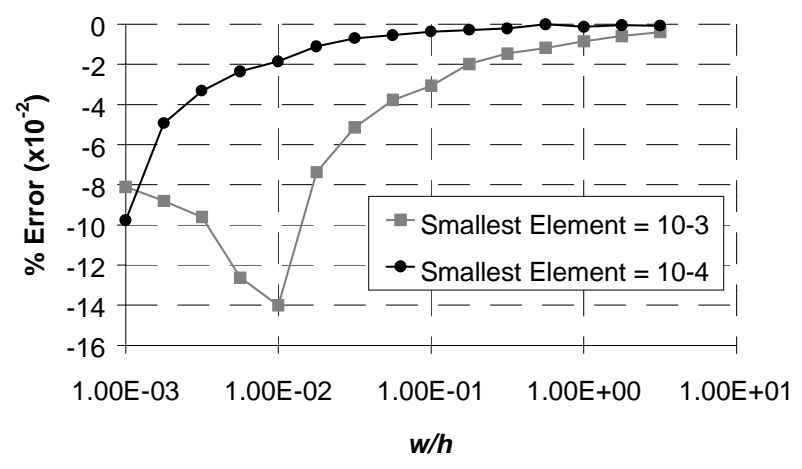

Figure 5 - Substrate $\varepsilon_{r}=\mathbf{4 . 2}$

The above graph shows that good accuracy can be obtained over nearly four decades of the width/height ratio. The computer processing time was less than $0.5 \mathrm{~s}$ for any of these values.

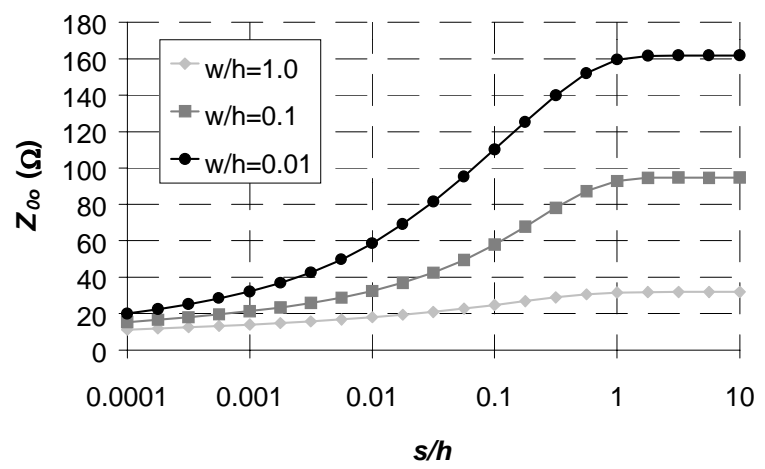

Figure 6 - Odd-mode impedance for different separations $(s / h)$ and widths $(w / h)$ 


\section{Coupled Coplanar Stripline}

Figure 6 shows the variation of the odd-mode impedance for the stripline shown in Figure 3.

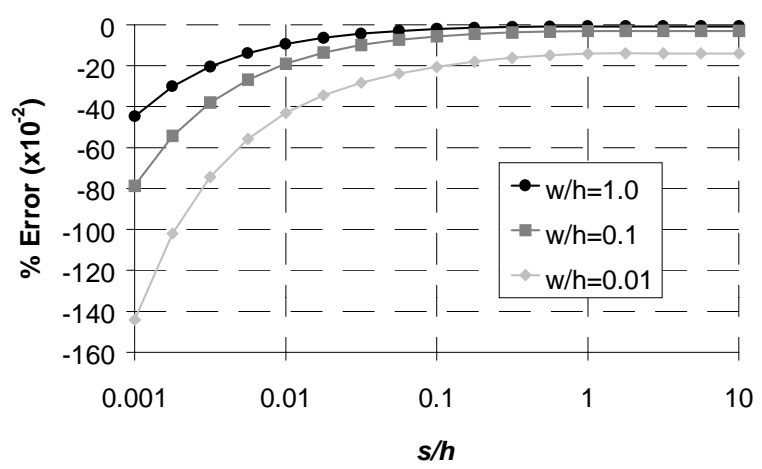

Figure 7 - \% error $\varepsilon_{r}=4.2$

Figure 7 shows the \% error of the numerical calculation compared with the exact values given by equation (7) using $10^{-3}$ as the smallest element. The maximum processing time was less then $0.5 \mathrm{~s}$. The maximum error can be reduced by decreasing the smallest element. For a maximum error of $6.0 \times 10^{-2} \%$, a processing time of $5.1 \mathrm{~s}$ is required.

The results presented in Figure 7 offer a very stringent test for the numerical method because of the sharp corners separated by s. In the odd-mode configuration this effect is enhanced even more because the tracks are of opposite polarity. This numerical validation is considered to be better then the results given by Bogatin et. al. ${ }^{[15]}$ for a pair of 'round' tracks (i.e. a parallel wire transmission line) using finite element software. In this latter case there are no singularities at the corners. $\mathrm{Li}$ and $\mathrm{Fujii}^{[16]}$ state that the boundary element method (to which MoM is related) is more accurate for stripline and microstrip than the finite element method.

\section{Surface Microstrip}

As previously mentioned there are no closed-form algebraic equations which are exact. But the discussion in the previous sections shows that the software can be made accurate, especially for practical purposes. Table 1 shows calculations for the configuration of Figure 1. Because the Green's Function involves a summation, and two capacitances $C$ and $C_{\text {air }}$ are required, processing times are now longer than those for stripline. The longest time was less than $4.5 \mathrm{~s}$ for a width of $3300 \mu \mathrm{m}$.

For coupled surface microstrip, two thick tracks of $3300 \mu \mathrm{m}$ requires a processing time of $5.1 \mathrm{~s}$. The separation does not affect the time.

\section{PRACTICAL RESULTS}

In order to verify the practical performance of the field solving boundary element method, the authors commissioned production of a set of samples. During a six month period in 1998, over 1500 different printed circuit board tracks were manufactured.

This sample consisted of both stripline and microstrip differential structures in surface and embedded configurations. Two types of coupled structures were included; edge-coupled and boardside-coupled. The track dimensions ranged from $75 \mu \mathrm{m}$ to $1000 \mu \mathrm{m}$ in width, with differential separations of 1 track width to 4 track widths using base copper weights of $1 / 2 \mathrm{oz}, 1 \mathrm{oz}$ and $2 \mathrm{oz}$. The resulting differential impedances ranged from $80 \Omega$ to $200 \Omega$.

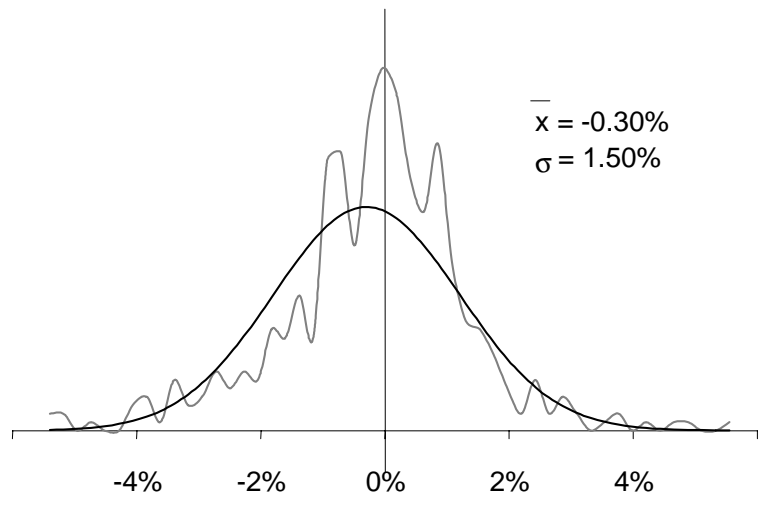

Figure 8 - Distribution of differences between predicted and measured values for stripline

Test samples were produced by three independent UK printed circuit board manufacturers ${ }^{[17]}$ and the differential impedances were electrically measured by TDR at Polar Instruments using a CITS500s Controlled Impedance Test System.

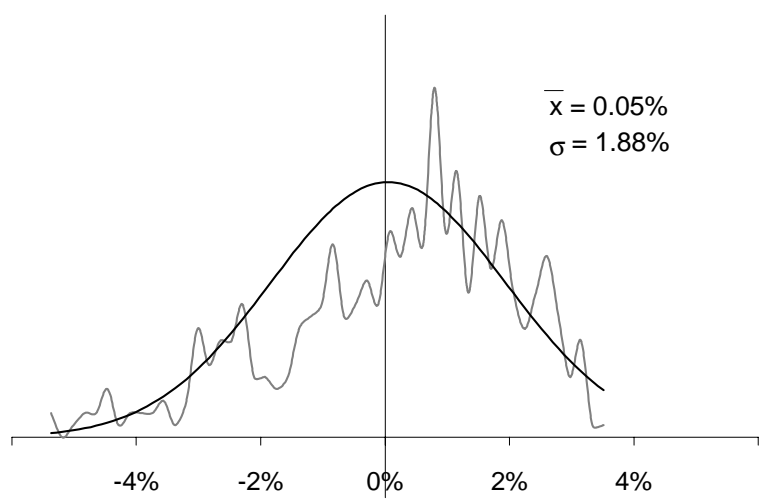

Figure 9 - Distribution of differences between predicted and measured values for embedded microstrip

After electrical measurement, the samples were returned to the manufacturers for microsection analysis to determine the actual physical mechanical dimensions. 
The calculated impedance was predicted from the mechanical microsection data and a derived value of relative permitivity, $\varepsilon_{r}$, of the FR-4 material. Results ${ }^{[18]}$ were analysed and comparisons of the electrically measured and the theoretically calculated results are presented in Figure 8 and Figure 9.

\section{DISCUSSION}

Accuracy of the electrical measurements is estimated at $1 \%$ to $2 \%$. This depends upon the impedance value and the quality of the interconnection between the test equipment and the test sample. Test samples were designed to be electrically balanced, but the manufacturing process will obviously not produce perfectly balanced traces.

Microsection dimensions have an estimated accuracy of $1 \%$, however the model assumes symmetry and this will introduce a further small averaging error estimated at $1 \%$. The total uncertainty in the experimental results is therefore estimated at $3 \%$ to $4 \%$. Figure 8 and Figure 9 show mean deviations of less than $0.5 \%$ with standard deviations of less than $2 \%$.

These practical results clearly show that the differences between the measured electrical results and the numerically calculated results are well within the estimated uncertainty of the measurement method.

\section{CONCLUSION}

The authors have shown that the early methods for calculating controlled impedance can now be used on desktop PC's. The accuracy is as good as, if not better than, the published algebraic equations. The processing times are less than 10 s which are acceptable in most cases.

Furthermore the number of configurations can be extended and trade cross-sectional profiles can be readily incorporated.

\section{REFERENCES}

1 Wadell, Brian C - Transmission Line Design Handbook Artech House 1991

2 IPC-2141 - Controlled Impedance Circuit Boards and High-Speed Logic Design, April 1996

3 Cohn, Seymour B. - Characteristic Impedance of the Shielded-Strip Transmission Line

IRE Trans MTT-2 July 1954 pp52-57
4 Abramowitz,Milton and Irene A Stegun - Handbook of Mathematical Functions, Dover, New York 1965

5 Hilberg, Wolfgang - From Approximations to Exact Relations for Characteristic Impedances. IEE Trans MTT-17 No 5 May 1969 pp259-265

6 Hart, Bryan - Digital Signal Transmission

Pub: Chapman and Hall 1988

7 Harrington, Roger F - Field Computation by Moment Methods, Pub: MacMillan 1968

8 CITS25 - Differential Controlled Impedance Calculator Polar Instruments Ltd, http://www.polar.co.uk, 1998

9 Sadiku, Matthew N O - Numerical Techniques in Electromagnetics, Pub: CRC Press 1992

10 Silvester P P - Microwave Properties of Microstrip Transmission Lines. IEE Proc vol 115 No 1 January 1969 pp43-48

11 Silvester P P \& Ferrari R L - Finite Element for Electrical Engineers Pub, Cambridge university Press 1983

12 Brebbia, C A - The Boundary Element Method for Engineers, Pub: Pentech Press 1980

13 Paris, Federico and Canas, Jose - Boundary Element Method : Fundamentals and Applications

Pub: Oxford University Press 1997

14 Kobayashi, Masanori Analysis of the Microstrip and the Electro-Optic Light Modulator

IEEE Trans MTT-26 No 2 February 1979 pp119-127

15 Bogatin, Eric; Justice, Mike; DeRego, Todd and Zimmer, Steve - Field Solvers and PCB Stack-up Analysis: Comparing Measurements and Modelling IPC Printed Circuit Expo 1998 paper 505-3

16 Li, Keren and Fujii, Yoichi - Indirect Boundary Element Method Applied to Generalised Microstrip Analysis with Applications to Side-Proximity Effect in MMICs IEE Trans MTT-40 No 2 February 1992 pp237-244

17 The authors wish to acknowledge the assistance of Kemitron Technologies plc, Stevenage Circuits Ltd and Zlin Electronics Ltd.

18 Surface microstrip results were yet to be completed at the submission date for this paper. 